\title{
Metabonomics: on the Road to Detect Diagnostic Biomarkers in Endemic (Balkan) Nephropathy. Evaluation in a Retrospective Pilot Project
}

Duquesne $\mathbf{M}^{1}$, Goossens $\mathbf{C}^{1}$, Dika $\check{Z}^{2}$, Conotte $\mathbf{R}^{1}$, Nortier $\mathrm{J}^{3}$, Jelaković $\mathrm{B}^{2}$ and Colet $\mathrm{JM}^{1 *}$

${ }^{1}$ University of Mons, Department of Human Biology \& Toxicology, 20 Place du Parc, 7000 Mons, Belgium

${ }^{2}$ School of Medicine, University of Zagreb, Department for Nephrology, Arterial Hypertension, Dialysis and Transplantation, University Hospital Center Zagreb, Zagreb, Croatia

${ }^{3}$ Department of Nephrology, Erasme Hospital, Université Libre de Bruxelles, 808 Route de Lennik, B-1070 Brussels, Belgium

\begin{abstract}
Introduction: Endemic (Balkan) Nephropathy is a chronic renal disease mainly affecting rural populations in the valleys of the Danube. In the absence of renal replacement therapy, it leads to fatal kidney failure and is significantly associated with upper urothelial carcinoma. Bread poisoning with aristolochic acids is now widely accepted. The source of this toxic substance is considered to be Aristolochia clematitis, a perennial plant that invades farming fields. The poisoning with aristolochic acids was suggested when clinical and histopathological changes similar to those observed in the Balkan patients were reported in several cases of nephropathy in Belgian patients unintentionally exposed to aristolochic acids during a Chinese herbs diet. Those clinical and histopathological features were then reproduced in laboratory experimental models.
\end{abstract}

Methods: Using metabonomics, an emerging dynamic technique that allows an effective mapping of alterations in endogenous metabolites levels in biofluids and tissues, we evaluated early signs of renal toxicity from extra urine samples collected in a rat model of intoxication with aristolochic acids.

Results: Changes in urine composition were consistent with a proximal tubular damage, most likely initiated by a mitochondrial default and an inappropriate response to oxidative stress. The same metabonomic approach was applied to surplus of urine samples collected from Belgian and Croatian patients in clinical and epidemiological studies, respectively. It allowed a clear discrimination of the Belgian patients from a database of healthy volunteers. On the other hand, a trend to discrimination was noticed when comparing urine samples collected from individuals living in Croatian endemic regions as compared to Croatian non endemic villages. Finally, when included in the same analysis, both Belgian and Croatian patients displayed similar urine metabolic signatures, suggesting a common etiology of both diseases.

Keywords: Endemic (Balkan) nephropathy; Aristolochic acid nephropathy; Aristolochic acids; Toxicity; Metabonomics; Epidemiology

Abbreviations: AAN: Aristolochic Acid Nephropathy; EN Endemic (Balkan) Nephropathy; CHN: Chinese Herb Nephropathy; ${ }^{1}$ H-NMR: Proton Nuclear Magnetic Resonance; TSP: Trimethylsilyl Propionic Acid; PCA: Principal Component Analysis; PLS-DA: Partial Least Squares Discriminant Analysis; MDRD: Modification of Diet in Renal Disease; CKD: Chronic Kidney Disease

\section{Introduction}

Since the 1950's, an unique kidney disease called Endemic (Balkan) Nephropathy (EN) is reported in Bosnia, Bulgaria, Croatia, Romania, and Serbia, countries located at South East part of Europe on the Balkan. It mainly affects rural populations in the valleys of the Danube tributaries and damages firstly the tubulo-interstitial sections of the kidney, slowly following a chronic evolution and leading to fatal end-stage-renalfailure. In the past, in the absence of dialysis or transplantation, many patients died in uremia. In addition, it is significantly associated with the occurrence of upper urothelial carcinoma, malignant tumors of the urinary tract epithelium which are barely observed outside the areas at risk for EN (also called "endemic regions"). Until the 1990's, ochratoxin A was the most suspected as the environmental cause of $\mathrm{EN}$, a mycotoxin produced by fungi growing in barns and grain crops and favored by the moisture occurring in these areas due to flood. Although it was detected in a variety of foods, its role in EN has never convinced scientists because of the lack of reproducibility in experimental kidney disease models [1,2]. In 2006, the EU Committee on Food Safety concluded that there is no convincing evidence from human epidemiology to confirm the association between ochratoxin an exposure and the prevalence of endemic nephropathy or urothelial cancers [3]. On the other hand, since 1993, a new clinical entity originally called "Chinese Herbs Nephropathy" (CHN) has been reported in Belgium first and then across the world [4]. This nephropathy was initially seen in women who had ingested slimming capsules based on Chinese herbs containing elements of Aristolochia species [5] Extracts of ground roots contained aristolochic acids I and II (AAI and AAII), recognized as nephrotoxic agents and carcinogens. Clinical and histopathological similarities (tubular atrophy, paucicellular fibrosis with cortico-medullary gradient leading to progressive interstitial renal insufficiency, upper urinary tract cancer) between this Aristolochic Acid-induced Nephropathy (AAN) and EN were then highlighted and reproduced in laboratory experimental models [6,7]. Another common

*Corresponding author: Jean-Marie Colet, University of Mons, Department of Human Biology and Toxicology, 20 Place du Parc, 7000 Mons, Belgium, E-mail: jean-marie.colet@umons.ac.be

Received November 16, 2012; Accepted December 04, 2012; Published December 06, 2012

Citation: Duquesne M, Goossens C, Dika Ž, Conotte R, Nortier J, et al. (2012) Metabonomics: on the Road to Detect Diagnostic Biomarkers in Endemic (Balkan) Nephropathy. Evaluation in a Retrospective Pilot Project. J Cancer Sci Ther S18: 002. doi:10.4172/1948-5956.S18-002

Copyright: (c) 2012 Duquesne M, et al. This is an open-access article distributed under the terms of the Creative Commons Attribution License, which permits unrestricted use, distribution, and reproduction in any medium, provided the original author and source are credited. 
sign was the detection of specific DNA adducts of AA metabolites in the renal cortex tissue of poisoned subjects and exposed animals as well as fingerprint mutation of oncogene p53, A:T->T:A transversion [810]. All these data converge to the hypothesis of a food poisoning by AA as a cause of EN, hypothesis already formulated by Ivić in 1969 who observed that farmers from endemic villages in Serbia had higher risk to be exposed to AA and had ingested it very probably through poisoned bread [11]. His long time neglected idea was confirmed for the first time in Croatian EN patients [12]. Before the real public health problem, a large number of interdisciplinary researchers around the world have joined forces in the evaluation of clinico-epidemiological and toxicological AA exposure [8]. Despite recent advances, the pathophysiological mechanisms causing EN are still far from being completely understood today. In addition, the lack of reliable clinical and/or biological markers to recognize the early stages of the disease is a major barrier for its diagnosis and appropriate management of the patients.

Recently, introduced in toxicology, metabonomics is a large scale in vivo screening method to rapidly evaluate the metabolic state of an organism from a peripheral sample such as urine or blood [13,14]. Upon exposure to a toxic substance, a disruption of the cellular homeostasis occurs with intermediary metabolic alterations which are reflected in the extracellular fluid of the target tissues, as well as in blood and urine. It is rare that a single endogenous biomolecule can accurately reflect a particular toxic effect. But, if a large number of biomolecules can be measured simultaneously, the "metabolic signature" they constitute all together is probably more consistent and more intuitive than any single marker. Obtaining such overall biochemical information is made possible by Proton Nuclear Magnetic Resonance ( $\left.{ }^{1} \mathrm{H}-\mathrm{NMR}\right)$ that can simultaneously detect a bench of soluble molecules, together forming the metabolome, and generally having a molecular weight $<1000 \mathrm{D}$. ${ }^{1} \mathrm{H}-\mathrm{NMR}$ spectra of biological sample, however, are extremely complex due to the large amount of information they include and require bioinformatics tools for multivariate data analysis. The combination of NMR spectroscopic analysis and multivariate data analysis provides a unique tool in the detection of various diseases and toxicities. From such characteristic "metabolic signatures", specific biomarkers of diseases or toxicities can be next isolated. As an example, a proximal tubular injury is very early associated with increased urinary levels of $\beta$-hydroxybutyrate, trimethylamine, and dimethylamine [15]. The knowledge of the biochemical pathways involving such biomarkers allows further inferences into cellular pathways involved in the progression and recovery of the pathology. Few NMR-based metabonomic studies have been published so far on the nephrotoxic effects of AA in rats [16,17] and in humans [18]. Metabonomics studies showed that proximal tubule is the primary target of AA in rats. Hence, the objective of this restrospective pilot project was to evaluate the potential of metabonomics in assessing EN and AAN from human and animal urines, respectively collected in previous and independent epidemiological and experimental studies.

\section{Materials and Methods}

\section{Animals and treatment}

All animal experiments were approved by the local Ethical Committee for animal care of the Institution (Erasme Hospital/ Université Libre de Bruxelles). All urine samples used in this study were surplus from previous experiments which were frozen at $-80^{\circ} \mathrm{C}$. Briefly, 12 Wistar male rats (age, $4 \mathrm{wk}$; weight, approximately $100 \mathrm{~g}$; Elevage Janvier, France) were housed in the animal care facility of the
Faculty of Medicine, Free University of Brussels (Brussels, Belgium). Groups of four or five animals were caged in a temperature-, humidity-, and light-controlled environment. Animals were fed ad libitum a normal diet (Carfil Quality, Oud-Turnhout, Belgium) and were allowed free access to water throughout the study. Animals received daily subcutaneous injections of a mixture of AA (Acros Organics Co., Geel, Belgium) dissolved in polyethylene glycol 400 (Fluka Chemie, Buchs, Switzerland) and diluted in distilled water before subcutaneous injection (AA-treated group, $15 \mathrm{mg} / \mathrm{kg}, \mathrm{n}=6$ ) or the vehicle only (control group, $\mathrm{n}=6$ ) for 35 days. On day 35, rats from each group were anesthetized with ether and euthanized by decapitation. Urine samples were collected on pretest and on study days 3, 7, 14, 21 and 35 in metabolic cages, centrifuged at $1600 \mathrm{xg}$ for $15 \mathrm{~min}$ at $4^{\circ} \mathrm{C}$, and stored at $-80^{\circ} \mathrm{C}$.

\section{Human urine samples}

Cohort 1: Surplus of urine samples collected from 5 AAN patients (2 samples/ patient) collected at separate consultations in the Department of Nephrology at Erasme Hospital (Brussels, Belgium) were used in this study. All patients signed an informed consent form to provide some urine samples for the metabonomic study. These AAN patients were suffering from non-terminal Chronic Kidney Disease (CKD) according to the MDRD (Modification of Diet in Renal Disease) classification: at a stage III (estimated glomerular filtration rate between 30 and $60 \mathrm{ml} /$ $\mathrm{min} / 1.73 \mathrm{~m}^{2}$ ) for 4 of them and at a stage IV (estimated glomerular filtration rate between 15 and $30 \mathrm{ml} / \mathrm{min} / 1.73 \mathrm{~m}^{2}$ ) for one of them.

Cohort 2: Surplus of urine samples used in this study were collected during a epidemiological survey conducted in Croatian endemic and non-endemic villages were stored at $-80^{\circ} \mathrm{C}$ in the presence of glycerol. Samples were collected from a total of 144 Croatian residents (49 males aged from 19 to 74 yrs with an average of 47 yrs and 95 women aged from 19 to 90 yrs with an average of 55 yrs. Among those individuals, 91 were inhabitants of endemic regions and 53 residents of non-endemic area. Subjects were classified using modified WHO criteria. Under those criteria farmers were classified into four groups: "diseased", "suspected" of having EN "at risk" and "others", based on the following diagnostic data: a) positive family/household history of EN; b) low molecular weight proteinuria (alpha-1 microglobulin $>10 \mathrm{mg} / \mathrm{l}$ or alpha-1 microglobuline/creatinine $>14 \mathrm{mg} / \mathrm{g}$ ); c) serum creatinine $>132.6 \mu \mathrm{mol} / \mathrm{l} ; \mathrm{d}$ ) anemia (hemoglobin $<120 \mathrm{~g} / \mathrm{l}$ if male, $\mathrm{Hb}<113 \mathrm{~g} / \mathrm{l}$ if female); e) exclusion of other renal disease (including diabetes)). Subjects were considered "diseased" if they were positive for " $a+b+c+d+e$ ", or " $b+c+d+e$ ", or " $a+b+d+e$ "; "suspected" if they had combination of " $a+b$ " or " $b+d$ "; "at risk" if they were from family/ household with EN and "others" if they did not comply with any of the above requirements. Based on those criteria subjects were classified as follows into the subgroups: "EN patients" $(\mathrm{n}=3)$, subjects 'at risk' for EN ( $n=7)$, subjects 'suspected of having EN' $(n=22)$, and 'others' $(n=59)$. Written informed consent was obtained from all participants. All protocols and procedures were approved by the relevant IRBs in Croatia.

Cohort 3: Urine samples were collected from 90 Belgian healthy volunteers (checked by a medical doctor for absence of any renal disease) during a medical examination at the Hospital Notre-Dame in Tournai, Belgium). The cohort included 41 women and 49 men aged from 20 to 90 yrs. Each volunteer had previously signed an informed consent form in accordance with the medical Ethics Committee of the Institution. Those urine samples were used for the building of the control database. 
Citation: Duquesne M, Goossens C, Dika Ž, Conotte R, Nortier J, et al. (2012) Metabonomics: on the Road to Detect Diagnostic Biomarkers in Endemic (Balkan) Nephropathy. Evaluation in a Retrospective Pilot Project. J Cancer Sci Ther S18: 002. doi:10.4172/1948-5956.S18-002

Page 3 of 9

\section{${ }^{1} \mathrm{H}-\mathrm{NMR}$ experiments}

Urine samples from the various cohorts were centrifuged $(5 \mathrm{~min}$ at $5,000 \mathrm{xg}$ at $\left.4^{\circ} \mathrm{C}\right)$ to remove any solid debris. Aliquots $(400 \mu \mathrm{l})$ of the supernatant fractions were removed to microcentrifuge tubes and mixed with $200 \mu \mathrm{L}$ of $0.2 \mathrm{M}$ sodium phosphate buffer $(0.04 \mathrm{M}$ $\mathrm{NaH}_{2} \mathrm{PO}_{4}+0.2 \mathrm{M} \mathrm{Na}_{2} \mathrm{HPO}_{4}, \mathrm{pH} 7.0$ ) prepared in $80 / 20 \mathrm{H}_{2} \mathrm{O} / \mathrm{D}_{2} \mathrm{O}$. $1 \mathrm{mM}$ TSP (trimethylsilyl propionic acid) was used as an external reference. $550 \mu \mathrm{L}$ were transferred in $5 \mathrm{~mm}$-NMR tubes and analyzed by ${ }^{1} \mathrm{H}-\mathrm{NMR}$ spectroscopy on a $500 \mathrm{MHz}$ Avance Bruker NMR System (Bruker, Karlsruhe, Germany). Solvent suppression of residual water signals was achieved via the NOESYPRESAT pulse sequence, in which the residual water peak is irradiated during the relaxation delay ( 1.5 $\mathrm{sec})$ and during the mixing time $(0.1 \mathrm{sec})$. For each sample, ${ }^{1} \mathrm{H}-\mathrm{NMR}$ spectra were collected using 64 scans containing 54,832 data points at a spectral width of $10,330.6 \mathrm{~Hz}$ with an acquisition time of $2.7 \mathrm{sec}$ and a relaxation delay of $3.0 \mathrm{sec}$.

\section{Data analysis}

The ${ }^{1} \mathrm{H}-\mathrm{NMR}$ spectra were phased and baseline corrected using Mest Re Nova 5.2.0 software (Mestre Lab Research, Santiago de Compostela, Spain). Chemical shift was referenced to TSP and binned in sub regions of $0.04 \mathrm{ppm}$ sections. All the spectra of the present study were manually phased and baseline-corrected manually. ${ }^{1} \mathrm{H}-\mathrm{NMR}$ spectrum over the ranged $0.2-10.0$ was reduced to sub regions of equal width $(0.04 \mathrm{ppm})$ and the signal intensity in each region integrated. The region between 4.5 and 5.0 was excluded prior to any statistical analysis in order to remove variability in the suppression of the water resonance. Data was normalized in Mest Re Nova by dividing each integrated sub region by the total area of the spectrum to reduce any dilution effect. Output ASCII data were next imported to Microsoft Excel (Microsoft Office, 2003), mean-centered and then exported to SIMCAP + 12 (Umetrics AB, Sweden) for multivariate data analysis of NMR spectral data. Principal component analysis (PCA) supplies two types of graphs: 1) the "scores plot" showing the separation(s) between study groups (each point represents one single observation, in this case a ${ }^{1} \mathrm{H}-\mathrm{NMR}$ spectrum of a urine sample); 2) the "loadings plot" showing the distribution of the corresponding variables responsible for the separation(s) observed in the scores plot (each point corresponds to the mean chemical shift of a particular spectral sub region of 0.04 ppm width, and consequently to the corresponding urine metabolites).

\section{Results}

\section{Animal data}

Urine samples were collected in rats after 3, 7, 14, 21 and 35 days of exposure to AA. The effect of such a treatment on the urine ${ }^{1} \mathrm{H}-\mathrm{NMR}$ profile is shown on figure 1 . A visual examination shows that some spectral regions are completely altered due to the exposure to aristolochic acids. The most obvious changes are seen in the aliphatic spectral regions (from 0.05 to $4.5 \mathrm{ppm}$ ). On study day 7 , dramatic decreases in the urinary levels of succinate (singlet at $2.43 \mathrm{ppm}$ ) and TMAO (singlet, $3.27 \mathrm{ppm}$ ) were observed and accompanied by large increases in $\alpha$-ketoglutarate ( 2 triplets at 2.45 and $3.01 \mathrm{ppm}$ ) and TMA.

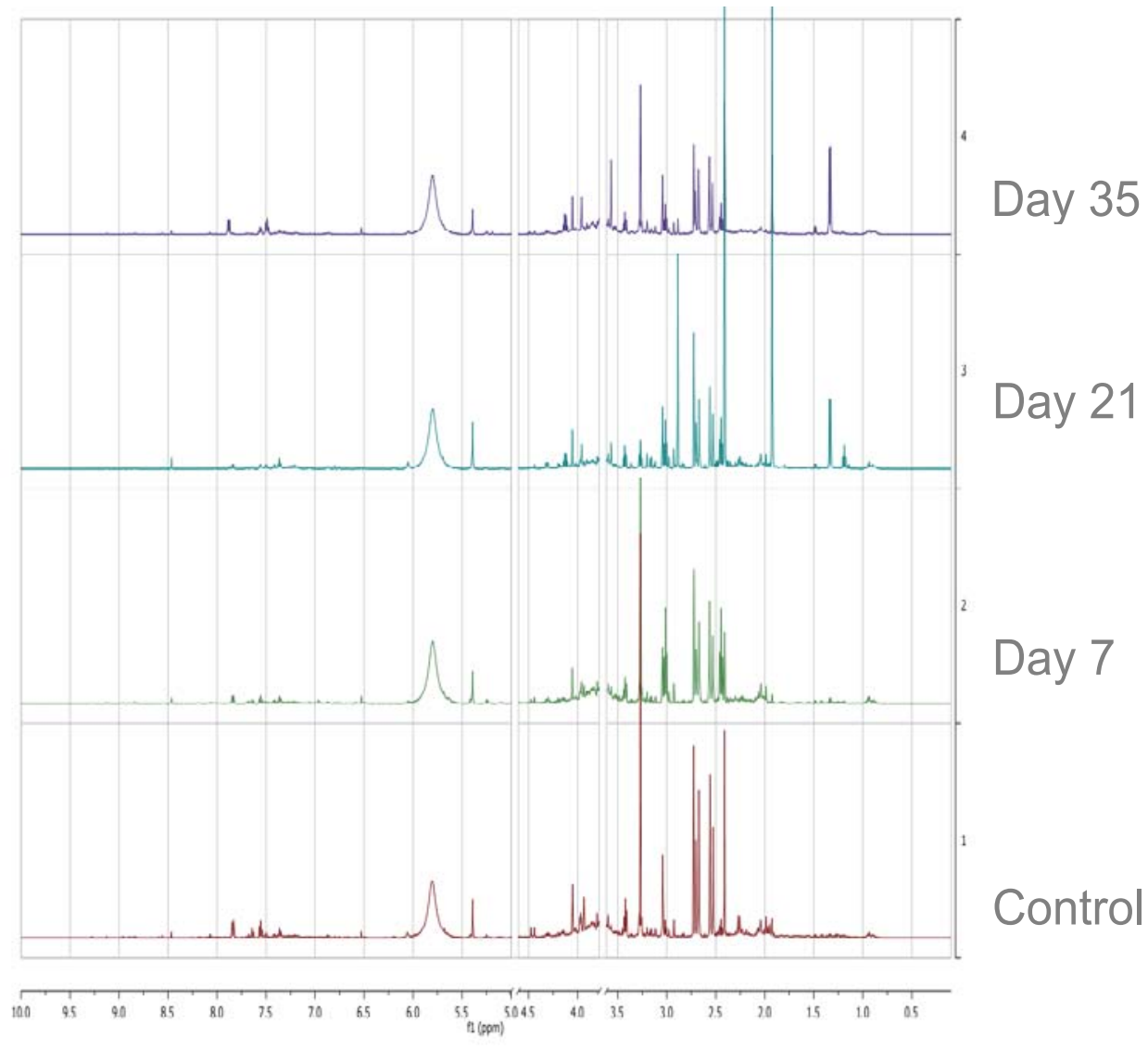

Figure 1: Time evolution of the ${ }^{1} \mathrm{H}-\mathrm{NMR}$ spectrum of urine samples collected from a rat exposed to aristolochic acids (s.c. $10 / \mathrm{mg} / \mathrm{kg}$ ) for 35 days. From bottom to top: urine samples collected before treatment, and on study Days 7, 21 and 35. 
Citation: Duquesne M, Goossens C, Dika Ž, Conotte R, Nortier J, et al. (2012) Metabonomics: on the Road to Detect Diagnostic Biomarkers in Endemic (Balkan) Nephropathy. Evaluation in a Retrospective Pilot Project. J Cancer Sci Ther S18: 002. doi:10.4172/1948-5956.S18-002

Page 4 of 9

At study days 21 and 35, those early changes seemed to progressively return to pre-test levels, however other peculiar changes were noticed, mainly corresponding to increases in lactate (doublet, $1.33 \mathrm{ppm}$ ) and DMA (singlet, $2.73 \mathrm{ppm}$ ).

In order to check whether those changes observed in that particular rat also occurred in the other animals exposed to aristolochic acid, the urine samples were separated from controls using PCA due to their distinct metabolic profiles. The scores plot (Figure 2) showed a clear separation between the NMR spectra of urine samples collected from the animals exposed to AA and those collected from the control rats. The corresponding loadings plot (Figure 3) clearly indicated that three major metabolites were mainly responsible for this separation between the two groups of animals: succinate, TMA, and TMAO. While the urine levels of succinate and TMA were largely increased, the excretion of TMAO was highly reduced. Besides those major changes, other alterations were also reported on the loadings plot and are gathered

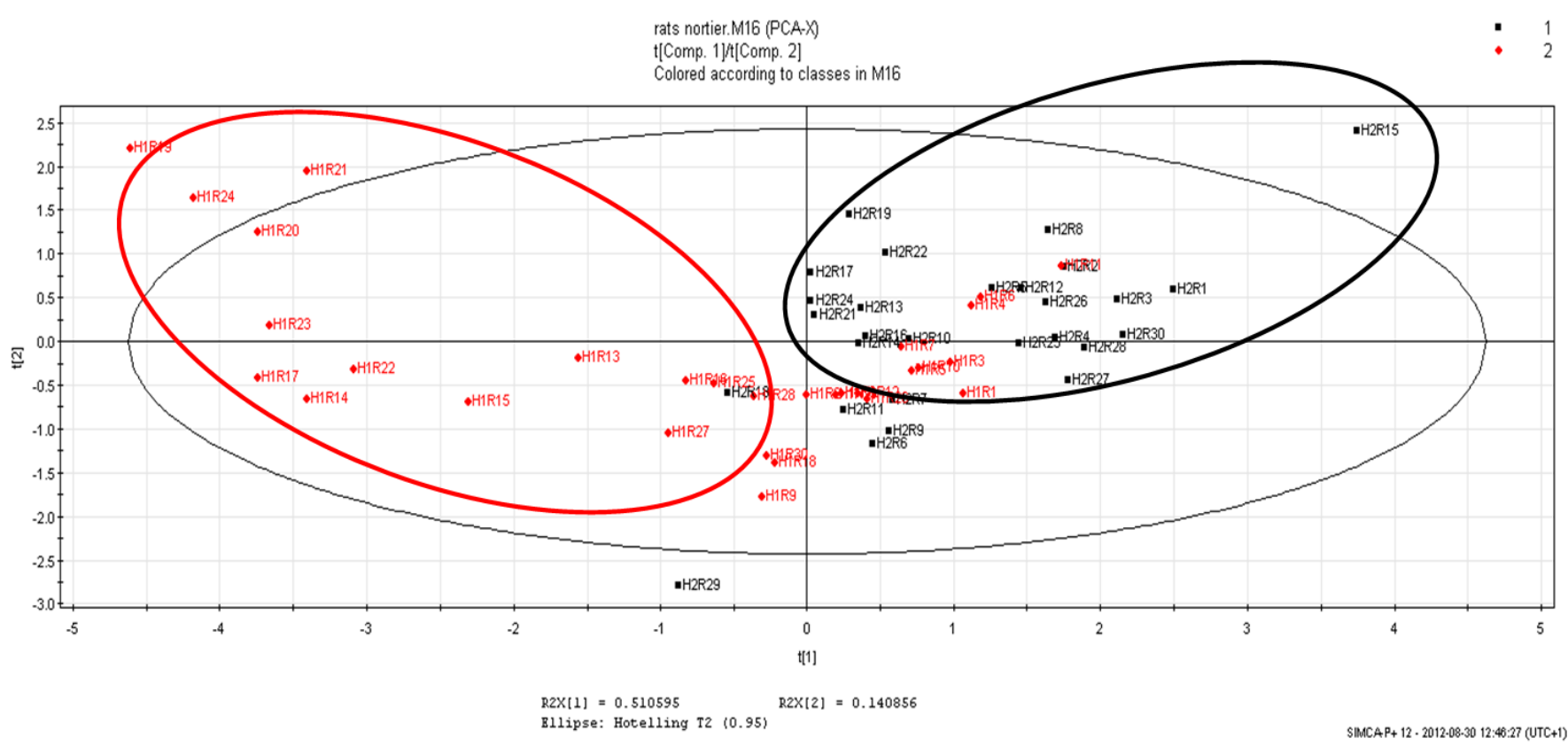

Figure 2: PCA (scores plot) of ${ }^{1} \mathrm{H}-\mathrm{NMR}$ spectra of urine samples collected from rats exposed to repeat doses of aristolochic acids (red diamonds) and control rats (black squares).

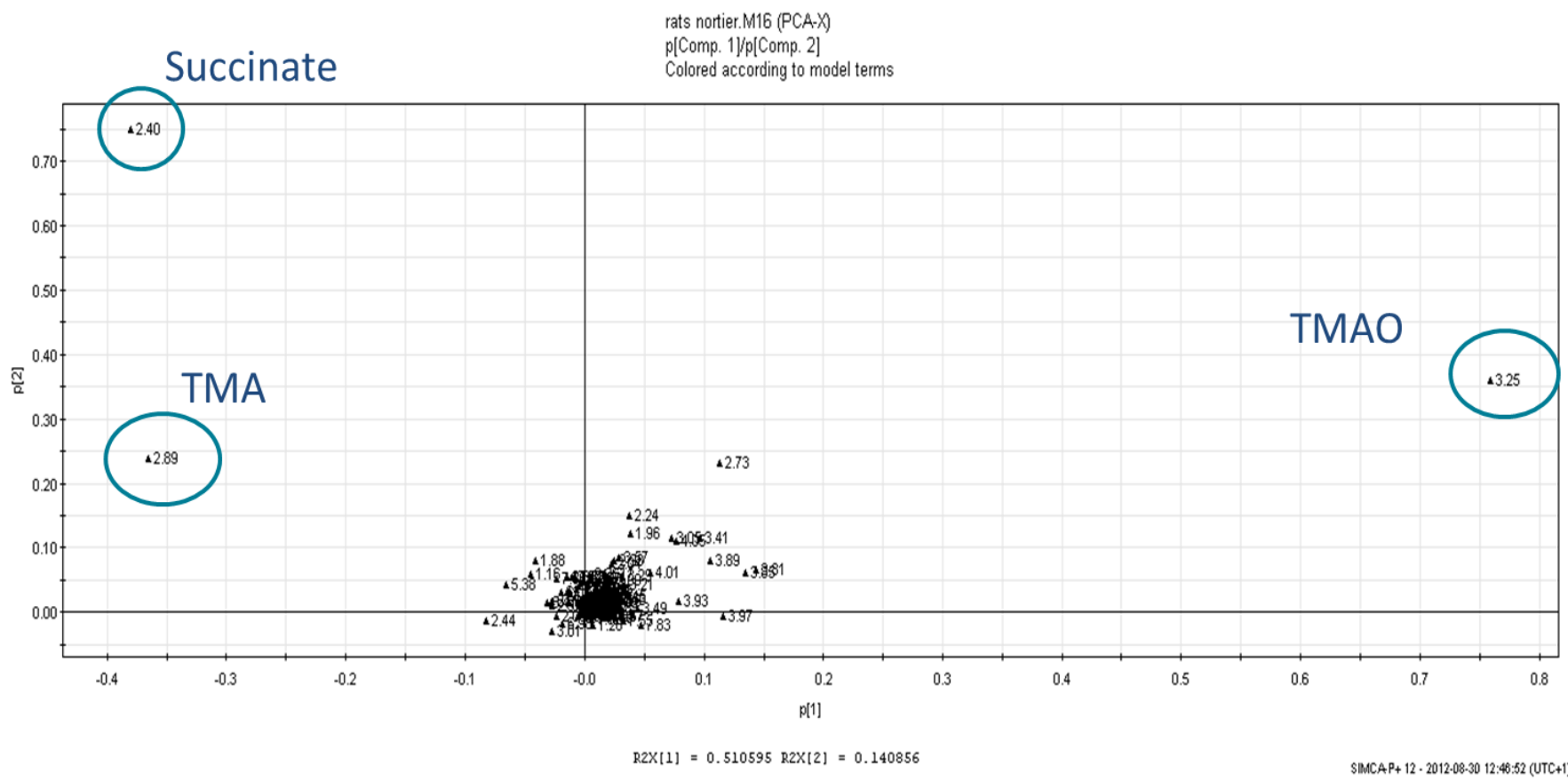

Figure 3: PCA (loadings plot) of ${ }^{1} \mathrm{H}-\mathrm{NMR}$ spectra of urine samples collected from rats exposed to repeat doses of aristolochic acids and control rats 
in table 1. Although quantitatively less important, these changes may have a higher biological value. Also, after exclusion of the descriptors corresponding to succinate, TMA, and TMAO, the same series of urine samples were next separated using PLS-DA, which offer the advantage of minimizing the variability within groups. The corresponding scores plot (Figure 4) revealed a better time separation of the samples over the study, showing that the greatest separation from controls was obtained somewhere between Day 14 and Day 21, followed by a trend towards a nearly complete recovery on Day 35.

\section{Human data}

Healthy volunteers: The ${ }^{1} \mathrm{H}-\mathrm{NMR}$ spectra of urine samples collected from 90 Belgian volunteers were submitted to unsupervised multivariate data analysis (PCA type). As anticipated, the corresponding scores plot (Figure 5) indicated a larger inter-individual variability amongst human subjects as compared to healthy animals. The most variability was observed in the urine levels of creatine, creatinine, and hippurate. A closer investigation revealed that the two major parameters causing such variability were the age (ranged from 20 to $90 \mathrm{yrs}$ ) and the gender of the subjects. Three individuals were also excluded because their urine samples were identified as outliers in the PCA due to abnormal levels of either glucose, or cis-aconitate, or acetaminophen-related resonances.

Belgian AAN-patients (previously known as "Chinese herb nephropathy"): A new PCA was performed on a subset of data gathering all samples collected from female Belgian volunteers and 10 urine samples received from 5 female AAN patients. The corresponding scores plot (Figure 6) showed a clear discrimination according to the first principal component amongst volunteer individuals. This separation was obviously based on the age and the optimal cut-off allowing the best separation was set at $65 \mathrm{yrs}$. The most influencing urine metabolites responsible for this separation were identified from the loadings plot (data not shown) as being glucose and creatinine. The second important observation was that the urine samples collected from AAN-patients were also nicely separated from the urines received from volunteers. This separation mainly occurred in the direction of the second principal component and was essentially driven by even higher urine levels of hippurate, creatinine, glucose and lower levels of citrate. Another relevant observation was the position of the urine samples from AAN patients in the left part of the sores plot, normally corresponding to females aged above 65 y.o., although those AAN patients were quite younger at the time of urine collection.

Croatian EN-patients (Endemic (Balkan) nephropathy, nowadays considered as an environmental form of AAN): The urine samples collected from people living in Croatian endemic $(n=91)$ and non-endemic $(n=53)$ regions were also analyzed by ${ }^{1} \mathrm{H}-\mathrm{NMR}$ spectroscopy and the NMR reduced data were submitted to a principal

\begin{tabular}{|l|}
\hline Urine markers \\
\hline Succinate \\
\hline Trimethylamine/Trimethylamine-N-oxide \\
\hline Creatinine \\
\hline Hippurate \\
\hline Glucose \\
\hline Lactate \\
\hline Alanine, valine, leucine, isoleucine \\
\hline a-ketoglutarate \\
\hline Dimethylamine \\
\hline
\end{tabular}

Table 1: List of urine metabolites mainly affected by the exposure to aristolochic acids. component analysis. The corresponding scores plot (Figure 7) showed a trend towards discrimination between both sub-populations (endemic vs. non-endemic). However, since significant age and gender influences were seen in the case of Belgian volunteers, the urine samples were also subdivided according to those parameters and an additional PCA was performed by comparing the urine NMR data from Croatian females living in the endemic regions and younger than 65 yrs with the Belgian AAN patients and healthy volunteers. The corresponding scores plot (Figure 8) not only showed a nice separation between AAN patients and female volunteers, as previously demonstrated, but in addition, some of the samples collected in EN endemic regions pretended to direct towards the same spatial location as Belgian AAN patients. This would suggest similar urine alterations in both diseased sub-populations as compared to the normal urine "metabonomic signature" established in healthy volunteers.

\section{Discussion}

While enjoying a positive image of "natural medicine", plant extracts consumed as food additives or as therapies pose a risk of adverse effects that is still largely ignored. Side effects of herbal medicines have often been reported in recent years, including tragic cases of intoxication with aristolochic acids, responsible for severe nephrotoxicity and urothelial carcinoma [4,5]. Aristolochic acids are naturally occurring at various concentrations in several species of Aristolochia and frequently discovered in Chinese, Japan and Indian herbs remedies. They are very potent nephrotoxins and have been linked to renal failure requiring transplantation in many cases worldwide. Importantly, they are accepted as carcinogens by the World Health Organization and according to the International Agency for Research on Cancer herbal remedies and natural mixtures containing plant species of the genus Aristolochia are considered carcinogenic to humans (Group 1) [19]. The clinical observation of some unusual cases of rapidly progressive renal failure has been identified for the first time in Belgium in 1992 [5]. The link with the consumption of a diet of plants containing Aristolochia fangchi was rapidly established [5,7]. The unwanted presence of this plant in various marketed mixtures comes from the confusion between two close Chinese vernacular names resulting in the replacement of Stephania tetrandra ("Han Fang-ji") by Aristolochia fangchi ("Guang Fang-ji") containing the highly toxic aristolochic acids [5]. Originally called "Chinese Herbs Nephropathy" (CHN), it is nowadays referred as "Aristolochic Acids Nephropathy" (AAN). It is a severe, progressive kidney disease, histologically characterized by an interstitial fibrosis very often complicated by urinary tract cancers. Histological appearance of AAN has many similarities with that of EN, a renal disease mainly distributed in Balkan villages located along the Danube and its tributaries. EN is characterized by a paucicellular chronic tubule-interstitial fibrosis with a slow progression to terminal uremia. Such as AAN, it is frequently complicated with upper urothelial carcinoma. The similarities between AAN and EN were mentioned in the early descriptions of cases referred to the CHN Belgian nephropathy, particularly the gradient of corticomedullary tubule-interstitial fibrosis, shared by both renal diseases [20]. More recently, DNA adducts with aristolactams were identified in the renal cortex and upper urothelial cancer of Croatian EN patients [10] and proposed as potential diagnostic biomarkers to confirm the disease [21]. It was proposed that $\mathrm{CHN}$ and $\mathrm{EN}$ are in fact the same entity caused with AA and differentiate in way of ingestion, while differences in clinical course could be explained by the difference in time in which toxic amount of AA was ingested [21,22].

Although the etiologic role of AA is now widely accepted by the 
Citation: Duquesne M, Goossens C, Dika Ž, Conotte R, Nortier J, et al. (2012) Metabonomics: on the Road to Detect Diagnostic Biomarkers in Endemic (Balkan) Nephropathy. Evaluation in a Retrospective Pilot Project. J Cancer Sci Ther S18: 002. doi:10.4172/1948-5956.S18-002

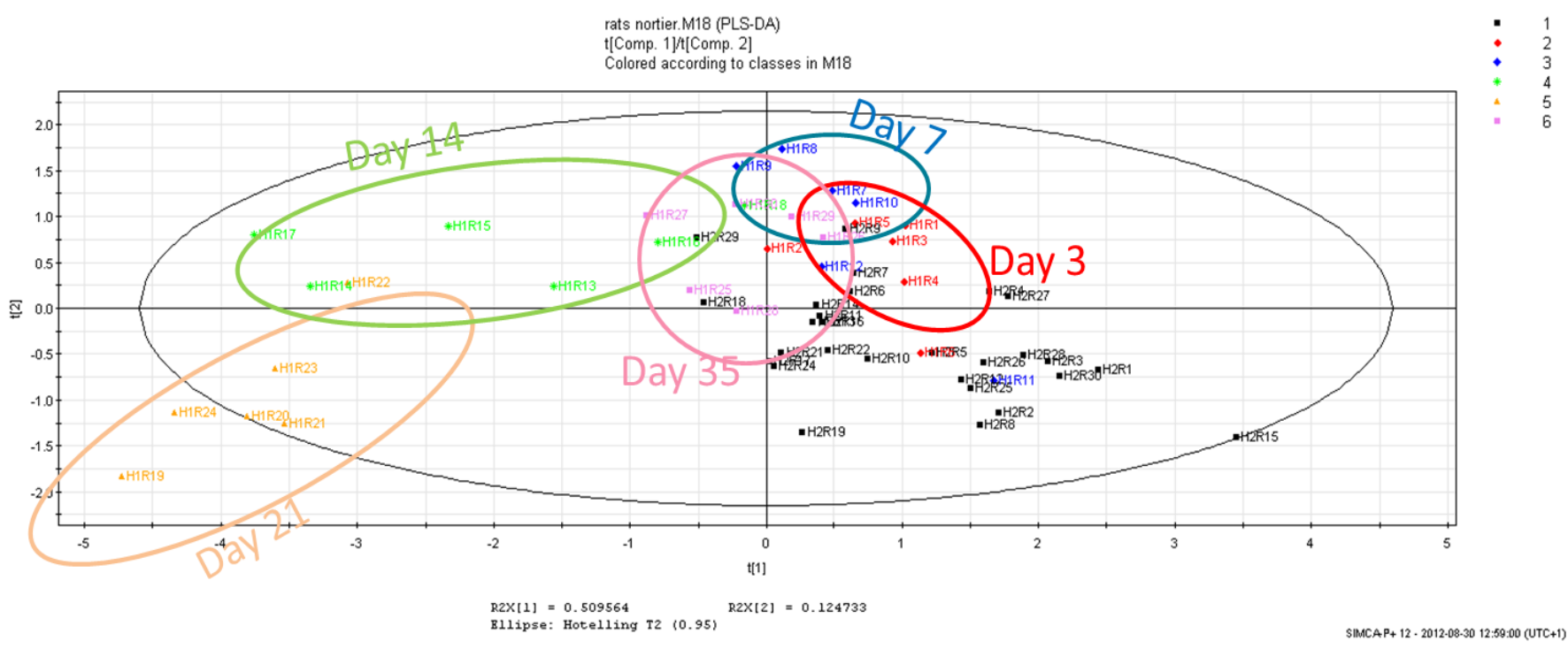

Figure 4: PLS-DA of ${ }^{1} \mathrm{H}-\mathrm{NMR}$ spectra of urine samples collected from control and AA-exposed rats: Time evolution.

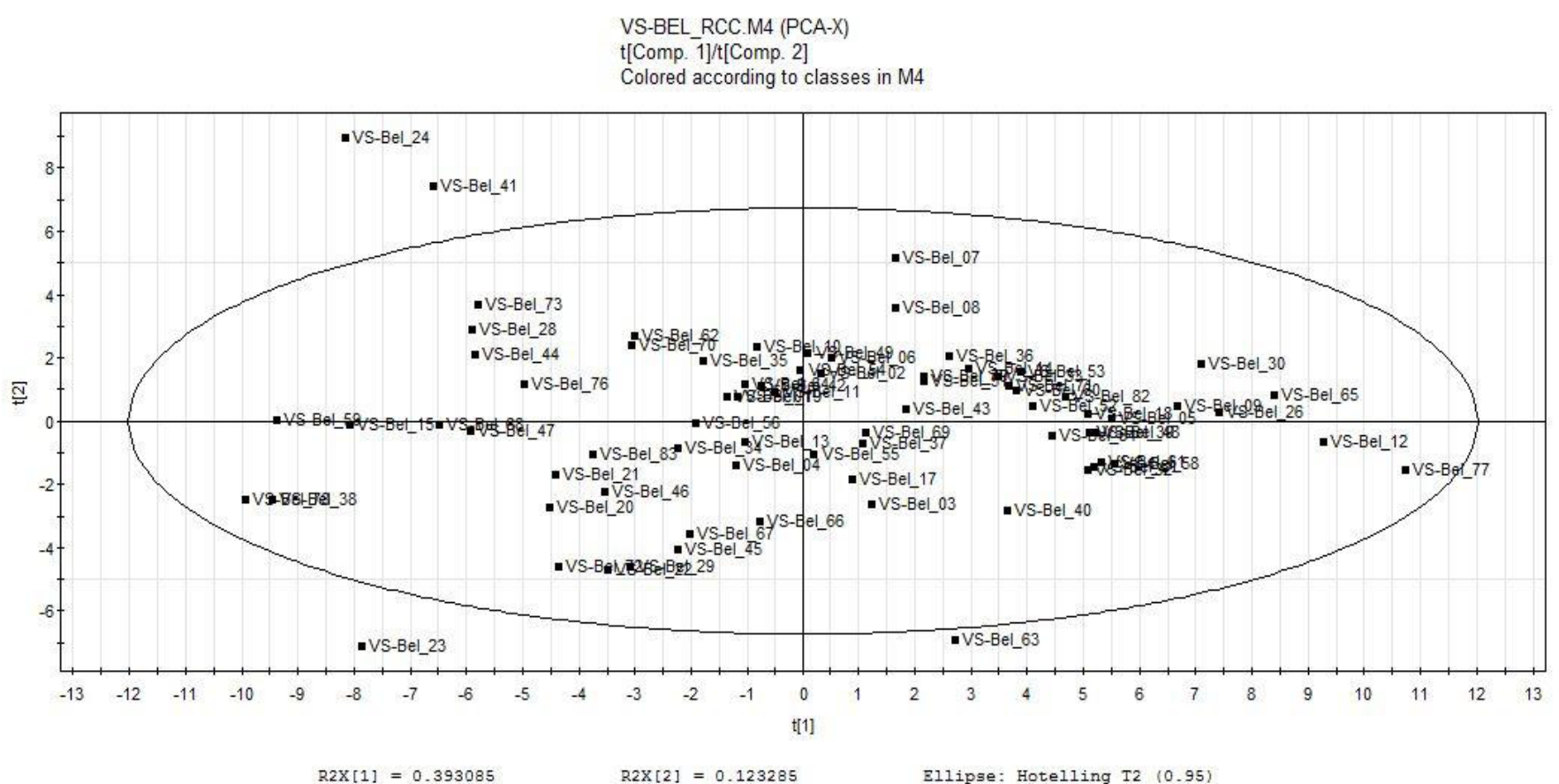

SIMCA-P+ $12-2012-09-05$ 15:08:25 (UTC+1)

Figure 5: PCA (scores plot) of ${ }^{1} \mathrm{H}-\mathrm{NMR}$ spectra of urtine samples collected from Belgian healthy volunteers.

medical and scientific communities from the findings in epidemiological and clinical studies, the mechanisms of toxicity are only partially resolved and access to diagnostic and prognostic biomarkers of AAN is still expected. Among others, an experimental model of AAN was developed by our group by daily subcutaneous injections of AA (10 $\mathrm{mg} / \mathrm{kg}$ ) in Wistar male rats [23]. AA-exposed animals developed chronic renal failure, tubular atrophy, mononuclear inflammatory infiltrate and interstitial renal fibrosis within 35 days.

Early predictive biomarkers of acute and chronic renal damages are essential to identify nephrotoxicity in preclinical model species and, of course, in human patients. To identify such predictive biomarkers that could recognize AAN early as well as aid in a better mechanistic understanding of this disease, we performed a retrospective metabonomics evaluation of frozen urine samples collected in epidemiological studies as well as from the experimental rat model described above. Metabonomics identifies metabolic signatures reflecting the global biochemical alterations occurring in a particular disease or following the exposure to a toxic substance. It is definitely more promising than the currently available clinical methods which are based on the detection of one single marker. From those complex 
Citation: Duquesne M, Goossens C, Dika Ž, Conotte R, Nortier J, et al. (2012) Metabonomics: on the Road to Detect Diagnostic Biomarkers in Endemic (Balkan) Nephropathy. Evaluation in a Retrospective Pilot Project. J Cancer Sci Ther S18: 002. doi:10.4172/1948-5956.S18-002

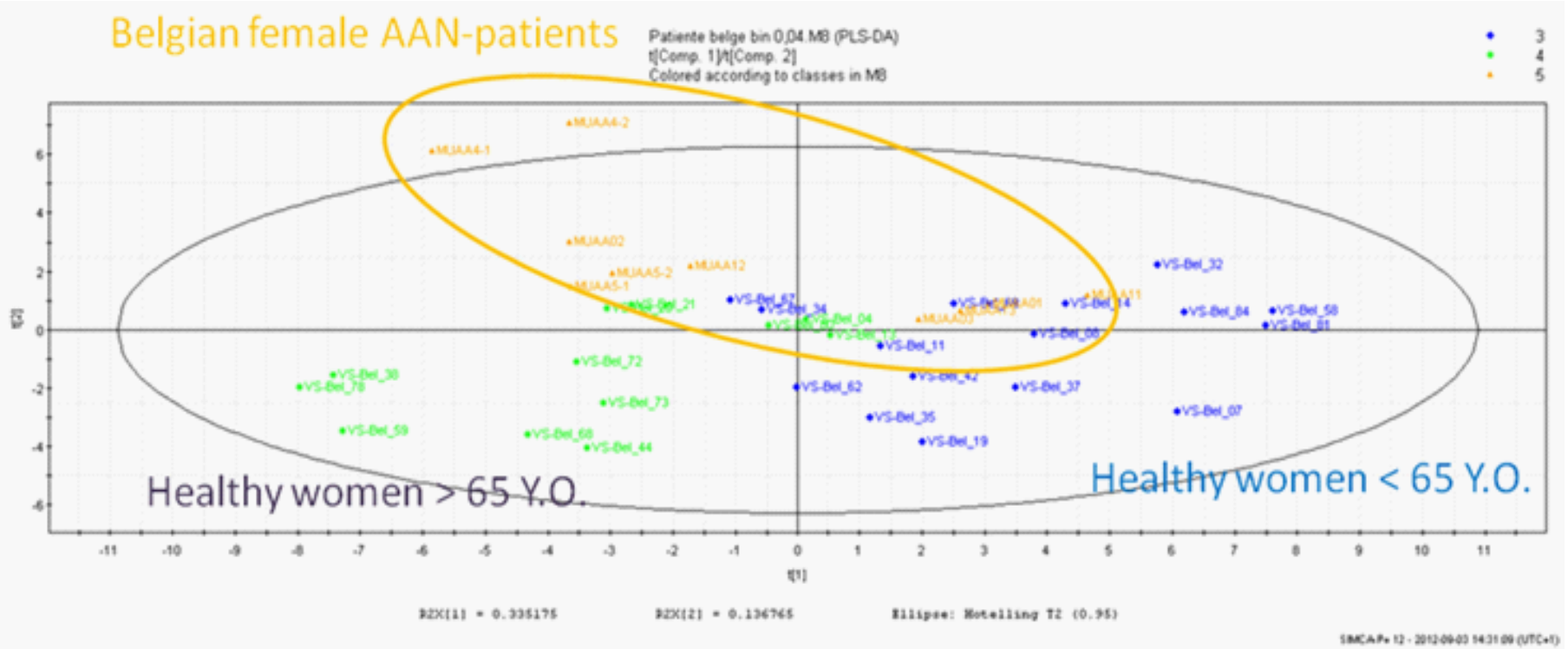

Figure 6: PLS-DA (scores plot) of ${ }^{1} \mathrm{H}-\mathrm{NMR}$ spectra of urine samples collected from healthy female Belgian volunteers (older than $65 \mathrm{y} .0$. green diamonds / younger than 65y.o. blue diamond's) and from 5 Belgian female AAN patients (yellow triangles).

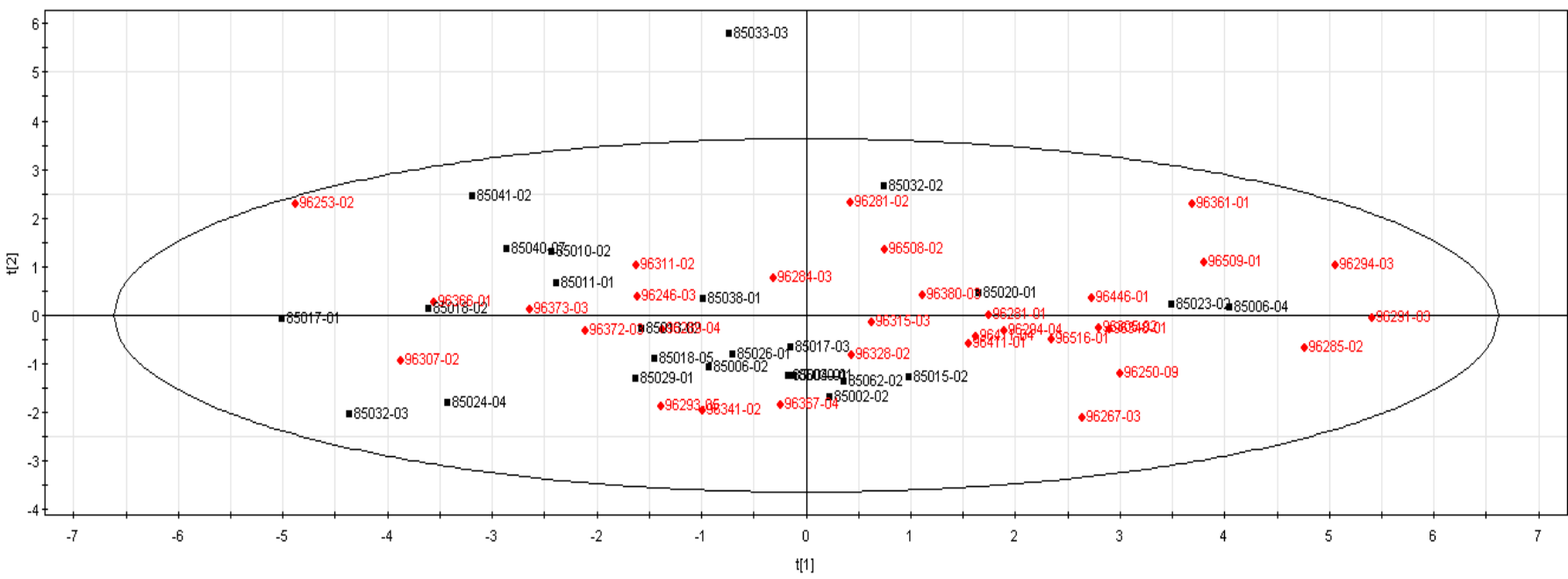

Figure 7: PCA (scores plot) of ${ }^{1} \mathrm{H}-\mathrm{NMR}$ spectra of urine samples collected from 91 inhabitants of endemic regions (red diamonds) and 53 inhabitants of non-endemic regions (black squares).

metabonomic signatures, a series of disease-specific biomarkers can be isolated which are expected not only to serve as early diagnostic tools but also to predict responses to treatment or drugs adverse effects.

In the present pilot study, surplus of human urines were thus received 1) from 5 Belgian women suffering from AAN due to consumption of diet pills inadvertently contaminated with AA, 2) from an epidemiologic study conducted in Croatian villages located in endemic (91 inhabitants) and non-endemic (53 inhabitants) regions, and 3) from 90 healthy Belgian volunteers. Surplus of urines collected from rats exposed to repeat doses of AA were also used. All urine samples were analyzed by ${ }^{1} \mathrm{H}-\mathrm{NMR}$ spectroscopy and the NMR data were further reduced in sub-regions of $0.04 \mathrm{ppm}$ width which were finally integrated to obtain numerical values. Multivariate data analysis was next applied to rapidly identify potential separation between groups and the urine metabolites responsible for the discrimination.
In the experimental model, the metabonomics approach was able to clearly discriminate AA-exposed rats from controls. The most important changes due to AA exposure were increases in urine levels of succinate and changes in the TMA/TMAO ratio, which could reflect alteration in the Krebs cycle activity and a response to oxidative stress and/or osmoprotection, respectively. Another group has previously highlighted the role of mitochondrial impairment and lack of activation of antioxidants enzymes in the process of tubular toxicity of AA [24]. Although less influencing, other metabolic changes were also noticed, including higher urine levels of glucose, lactate, and various amino acids accompanied with decreases in creatinine and hippurate. Those metabolic changes were already reported by several reports from the COMET consortium [25-27] that performed toxicological metabonomic studies using a large number of prototypical toxins $(\mathrm{n}=147)$ and pathophysiological conditions. The resulting 


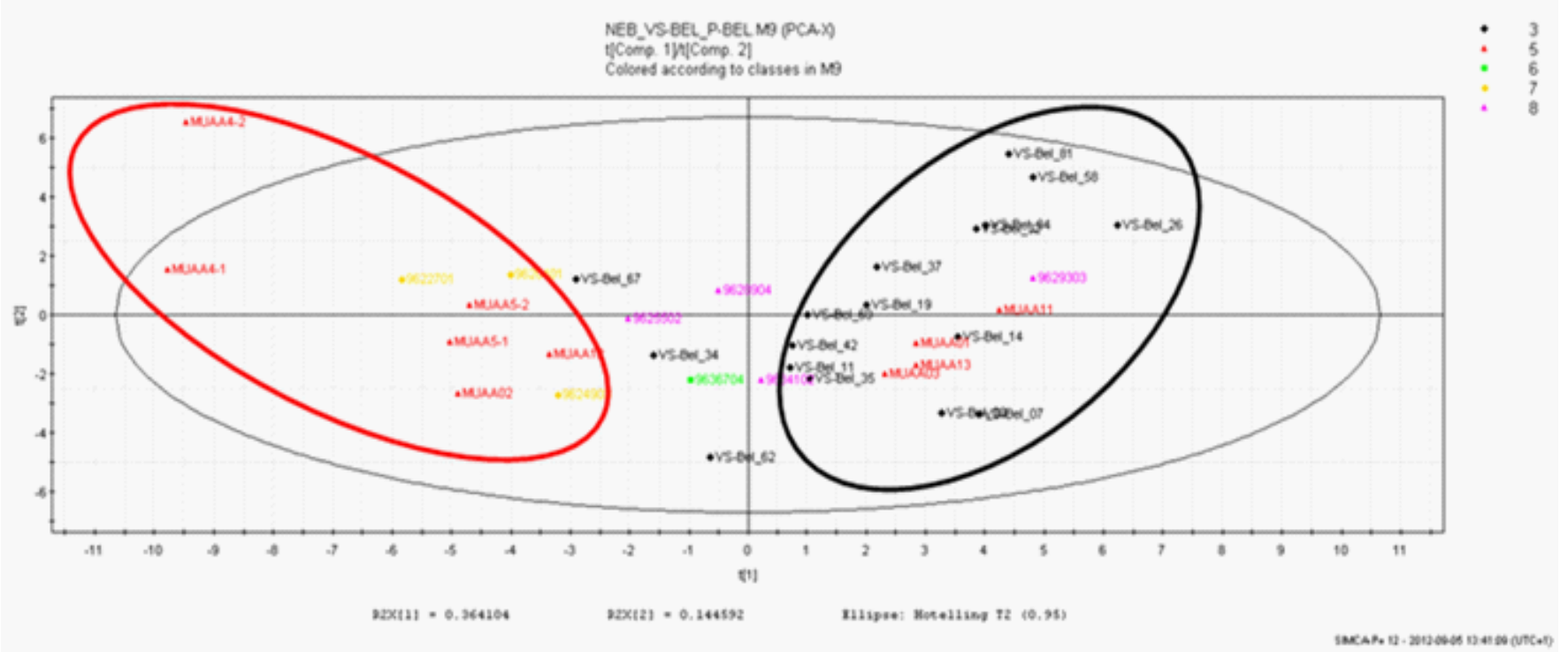

Figure 8: PCA (scores plot) of ${ }^{1} \mathrm{H}-\mathrm{NMR}$ spectra of urine samples collected from female subjects: Belgian healthy women (black squares), Belgian AAN patients (red triangles); Croatian EN-patients (green circle); EN-suspected Croatian women (yellow diamonds), EN-at risk Croatian women (magenta diamonds).

metabonomics data were used to generate an expert system for the prediction of rodent kidney and liver toxicity. According to those reports, the metabolic alterations identified in our rat experimental model of AAN are in agreement with an injury of the sections S1 and $\mathrm{S} 3$ of the renal proximal tubules sections which are less active in reabsorbing glucose and amino acids, and in secreting hippurate. The reduction in creatininuria most likely reflects a default in the renal glomerular filtration function. Interestingly, those changes occurred within the first days of AA-exposure but rapidly disappeared upon retrieval of the drug. This demonstrated the reversibility of the renal tubular injury.

As expected, in comparison to the animal model, the metabonomic approach in humans is complicated by a larger interindividual variability. In our pilot study, the most variable urine metabolites among healthy volunteers were creatinine, hippurate, and creatine. Such differences were already reported by others $[28,29]$ and associated to diurnal fluctuations and diet effects. In our case, those changes were also attributed to age (creatinine and hippurate) and gender (creatine or creatinine) effects. In order to minimize such interindividual variations, two databases of Belgian healthy volunteers were created by separating males from females and within both subpopulations an optimal age cut-off was determined and fixed at 65 yrs. Urine NMR data obtained from Belgian AAN-patients were next confronted to the female healthy volunteer database. This comparison allowed us to clearly discriminate the AAN-patients based on dramatic reduction in the excretion of creatinine and hippurate as well as increased urine levels of glucose and lactate. Those changes were quite confusing because some of them were similar, although in a more severe extent, to those discriminating healthy volunteers on the age basis. One interesting observation though was the location of the AAN patients (younger than 65 yrs at the time of urine collection) in the PCA region normally allocated to women older than $65 \mathrm{yrs}$. It is most likely that those urine metabolic markers reflect a progressive alteration in the kidney function with age in the healthy volunteer population but, at higher concentrations, an abnormal renal activity in AAN patients as compared to their aged-matched controls.
Finally, the metabonomic approach was applied to a series of urine samples collected from people living in endemic and non-endemic Croatian villages. Although a trend to discrimination was noticed, the multivariate data analysis barely separated people living in endemic and non-endemic regions. Besides the natural inter-individual variability already observed in the Belgian population, another explanation could be the presence of unaffected subjects in the endemic regions while "others" and those "at risk", who were supposed to be more exposed to AA in the past than farmers from non-endemic villages but had no signs of the renal affection were included in the cohort from endemic villages. Lack of significance in difference might be also caused by the presence of so-called "sporadic forms" of EN diseased in the nonendemic subpopulation. Finally, maybe difference in time of exposure, period of time in which toxic amount of AA was ingested (high doses during weeks/months in Belgian patients vs. low doses over dozens of years in $\mathrm{EN}$ ) could contribute to difference in observation between Belgian and Croatian subgroups. Hence, the urine NMR data collected from Croatian women living in the endemic regions and aged less than 65 yrs were included in the predictive model of AAN (Belgian AAN patients $v s$. healthy volunteers). Some of the samples collected in endemic regions presented urine metabolic alterations similar to those recorded in Belgian AAN patients, suggesting a similar etiology. In a recent study, ${ }^{1} \mathrm{H}$-NMR-based metabonomics was applied to various populations in Romania and Bulgaria (people diagnosed with EN, individuals treated by hemodialysis, and subjects without overt renal disease) [18]. The authors concluded that this metabolomic analysis can be predictive of impending morbidity before conventional criteria can diagnose EN and potentially differentiate etiology of this nephropathy in different geographic foci across the Balkans.

In terms of public health, the history of AAN reminds us that all naturally occurring products are not necessarily safe. The uncontrolled access to such products on the Internet increases the risk of a worldwide contamination. Natural food additives and remedies should be submitted to the same controls as drugs regarding their efficacy and safety. A recent review of methods used in safety evaluation concludes that conventional assays should integrate new "omics" technologies (transcriptomics, proteomics, metabonomics) [30]. 
Citation: Duquesne M, Goossens C, Dika Ž, Conotte R, Nortier J, et al. (2012) Metabonomics: on the Road to Detect Diagnostic Biomarkers in Endemic (Balkan) Nephropathy. Evaluation in a Retrospective Pilot Project. J Cancer Sci Ther S18: 002. doi:10.4172/1948-5956.S18-002

\section{Acknowledgement}

The authors are grateful to Prof. Luce Vander Elst (Department of General, Organic and Biomedical Chemistry at the University of Mons, Mons, Belgium for her scientific support and access to ${ }^{1} \mathrm{H}-\mathrm{NMR}$ spectrometer, to Ante Cvitković M.D. Ph.D. (Institute for Public Health County Brodsko-Posavska, Slavonski Brod Croatia) for his help in conducting field work during the survey in Croatia.

The research in Croatia was supported by award 108-0000000-0329 from the Croatian Ministry of Science.

\section{References}

1. Stefanović V, Toncheva D, Atanasova S, Polenaković M (2006) Etiology of Balkan endemic nephropathy and associated urothelial cancer. Am J Nephro 26: 1-11.

2. Bamias J, Boletis J (2008) Balkan nephropathy: evolution of our knowledge. Am J Kidney Dis 52: 606-616.

3. Opinion of the Scientific Panel on Contaminants in the Food Chain on a request from the Commission related to ochratoxin A in food, adopted on 4 April 2006, The EFSA Journal 365: 1-56.

4. Depierreux M, Van Damme B, Vanden Houte K, Vanherweghem JL (1994) Pathologic aspects of a newly described nephropathy related to the prolonged use of Chinese herbs. Am J Kidney Dis 24: 172-180.

5. Vanherweghem JL, Depierreux M, Tielemans C, Abramowicz D, Dratwa M et al. (1993) Rapidly progressive interstitial renal fibrosis in young women: association with slimming regimen including Chinese herbs. Lancet 341: $387-$ 391.

6. Debelle FD, Vanherweghem JL, Nortier JL (2008) Aristolochic acid nephropathy: a worldwide problem. Kidney Int 74: 158-169.

7. Nortier JL, Martinez MC, Schmeiser HH, Arlt VM, Bieler CA, et al. (2000) Urothelial carcinoma associated with the use of a Chinese herb (Aristolochia fangchi). N Engl J Med 342: 1686-1692.

8. Grollman AP, Shibutani S, Moriya M, Miller F, Wu L, et al. (2007) Aristolochic acid and the etiology of endemic (Balkan) nephropathy. Proc Natl Acad Sci U S A 104: 12129-12134.

9. Stiborová M, Frei E, Arlt VM, Schmeiser HH (2008) Metabolic activation of carcinogenic aristolochic acid, a risk factor for Balkan endemic nephropathy. Mutat Res 658: 55-67.

10. Grollman AP, Jelaković B (2007) Role of environmental toxins in endemic (Balkan) nephropathy. October 2006, Zagreb, Croatia. J Am Soc Nephrol 18: 2817-2823.

11. Ivić M (1969) Etiology of endemic nephropathy. Lijec Vjesn 91: 1273-1281.

12. Hranjec T, Kovac A, Kos J, Mao W, Chen JJ, et al. (2005) Endemic nephropathy: the case for chronic poisoning by aristolochia. Croat Med J 46: 116-125.

13. Lindon JC, Nicholson JK, Holmes E, Antti H, Bollard ME, et al. (2003) Contemporary issues in toxicology the role of metabonomics in toxicology and its evaluation by the COMET project. Toxicol Appl Pharmacol 187: 137-146.

14. Lindon JC, Holmes E, Bollard ME, Stanley EG, Nicholson JK (2004) Metabonomics technologies and their applications in physiological monitoring, drug safety assessment and disease diagnosis. Biomarkers 9: 1-31.
15. Shockcor JP, Holmes E (2002) Metabonomic applications in toxicity screening and disease diagnosis. Curr Top Med Chem 2: 35-51.

16. Chen M, Su M, Zhao L, Jiang J, Liu P, et al. (2006) Metabonomic study of aristolochic acid-induced nephrotoxicity in rats. J Proteome Res 5: 995-1002.

17. Zhang X, Wu H, Liao P, Li X, Ni J, et al. (2006) NMR-based metabonomic study on the subacute toxicity of aristolochic acid in rats. Food Chem Toxico 44: 1006-1014.

18. Mantle P, Modalca M, Nicholls A, Tatu C, Tatu D, et al. (2011) Comparative ${ }^{1} \mathrm{H}-\mathrm{NMR}$ Metabolomic Urinalysis of People Diagnosed with Balkan Endemic Nephropathy, and Healthy Subjects, in Romania and Bulgaria: A Pilot Study. Toxins 3: 815-833

19. Grosse Y, Baan R, Straif K, Secretan B, El Ghissassi F, et al. (2009) A review of human carcinogens-Part A: pharmaceuticals. Lancet Oncol 10: 13-14.

20. Cosyns JP, Jadoul M, Squifflet JP, Van Cangh PJ, van Ypersele de Strihou C (1994) Urothelial malignancy in nephropathy due to Chinese herbs. Lance 344: 188.

21. Jelaković B, Karanović S, Vuković-Lela I, Miller F, Edwards KL, et al. (2012) Aristolactam-DNA adducts are a biomarker of environmental exposure to aristolochic acid. Kidney Int 81: 559-567.

22. De Broe ME (2012) Chinese herbs nephropathy and Balkan endemic nephropathy: toward a single entity, aristolochic acid nephropathy. Kidney In 81: 513-515.

23. Debelle FD, Nortier JL, de Prez EG, Garbar CH, Vienne AR, et al. (2002) Aristolochic acids induce chronic renal failure with interstitial fibrosis in saltdepleted rats. J Am Soc Nephrol 13: 431-436.

24. Pozdzik AA, Salmon IJ, Debelle FD, Decaestecker C, Van den Branden C, et al. (2008) Aristolochic acid induces proximal tubule apoptosis and epithelial to mesenchymal transformation. Kidney Int 73: 595-607.

25. Beckonert O, Bollard ME, Ebbels TMD, Keun HC, Antti H, et al. (2003) NMRbased metabonomic toxicity classification: hierarchical cluster analysis and k-nearest-neighbour approaches. Analytica Chimica Acta 490: 3-15.

26. Keun HC (2006) Metabonomic modeling of drug toxicity. Pharmacol Ther 109 92-106.

27. Lindon JC, Keun HC, Ebbels TM, Pearce JM, Holmes E, et al. (2005) The Consortium for Metabonomic Toxicology (COMET): aims, activities and achievements. Pharmacogenomics 6: 691-699.

28. Lenz EM, Bright J, Wilson ID, Morgan SR, Nash AF (2003) A 1H-NMR-based metabonomic study of urine and plasma samples obtained from healthy human subjects. J Pharm Biomed Anal 33: 1103-1115.

29. Walsh MC, Brennan L, Malthouse JPG, Roche HM, Gibney MJ (2006) Effect of acute dietary standardization on the urinary, plasma, and salivary metabolomic profiles of healthy humans1,2,3. Am J Clin Nutr 84: 531-539.

30. Ouedraogo M, Baudoux T, Stévigny C, Nortier J, Colet JM, et al. (2012) Review of current and "omics" methods for assessing the toxicity (genotoxicity, teratogenicity and nephrotoxicity) of herbal medicines and mushrooms. J Ethnopharmacol 140: 492-512. 